\title{
Biochemical Bone Markers for Early Detection of Osteopenia of Prematurity
}

\author{
Jahan $\mathrm{I}^{1}$, Mannan MA ${ }^{1}$, Dey AC ${ }^{1}$, Dey $\mathrm{SK}^{1}$, Choudhury SM${ }^{1}$, Shahidullah $\mathrm{M}^{1}$ \\ ${ }^{I}$ Department of Neonatology, Bangabandhu Sheikh Mujib Medical University, Dhaka, Bangladesh \\ e-mail: ismatjahandr@gmail.com
}

\begin{abstract}
Osteopenia of prematurity (OOP) imposes the risk of fractures and growth failure to premature infants. Studies have investigated the validity of biochemical markers of osteopenia but till date it is not established. So, this study was intended to examine the diagnostic performance of biochemical markers in early detection of osteopenia of prematurity. This prospective study was conducted in the Neonatal Intensive Care Unit (NICU), Department of Neonatology, Bangabandhu Sheikh Mujib Medical University during June 2013 to February 2014. A total of 100 premature infants with gestational age $\leq 34$ weeks were consecutively included over 9 months period. Serum alkaline phosphatase, serum calcium and serum inorganic phosphates were measured from 1 week of chronological age until corrected term age. At corrected term age, radiologic examination was done for the assessment of osteopenia. Of the enrolled infants, 36/78 (46\%) developed radiological evidence of osteopenia. Serum inorganic phosphate level was significantly less in osteopenic infants than non-osteopenic infants throughout first two months of life $(p<0.001)$. The area under ROC curve for serum inorganic phosphate was $85 \%(p=0.001)$. If the cut off value of serum inorganic phosphate was set at $3.6 \mathrm{mg} / \mathrm{dl}$, then a sensitivity of $86 \%$ and a specificity of $49 \%$ were obtained. Low serum inorganic phosphate at 3 weeks of life can be used as a marker for early detection of osteopenia of prematurity.
\end{abstract}

Key words: Osteopenia, Premature infants, Biochemical markers

\section{Introduction}

In the face of advancement in antenatal and perinatal care, every year, 15 million babies are born prematurely around the world of which more than 1 million die. ${ }^{1}$ However, the continuous developments in intensive care have led to a progressive decline in mortality. ${ }^{2}$ The achievement through an intensive care has not always ensured a favourable outcome of premature newborn. Osteopenia of prematurity (OOP) is an important co-morbidity which expose them to the risk of fractures and growth failure. ${ }^{3}$ Premature infants are at risk of suboptimal bone mineralisation in early life, as the later part of gestational period is vital for mineral accretion. ${ }^{4}$ Their bones mineral content at term corrected age has been found to be 25-70\% lower than in term. ${ }^{5}$ Most of the previous studies reported that incidence of osteopenia of prematurity was as high as up to
$75 \% .^{6,7}$ Radiologic evidence of osteopenia has been reported in $23 \%$ of very low birth weight infants and in $55 \%$ to $60 \%$ of extremely lowbirth weight infants. Fractures were reported in nearly $10 \%$ of VLBW infants at 36 to 40 weeks postmenstrual age. ${ }^{8}$ Possibility of declination of incidence is anticipated over past two decades because of nutritional management. ${ }^{9}$ Recent data support its existence especially in extremely low birth weight population. ${ }^{3}$ Bangladesh is among the top 10 countries with the highest numbers of preterm birth. ${ }^{1}$ Dealing prematurity related complications are the great challenges for the neonatal physicians. Proportion of osteopenia of prematurity was $28 \%$ according to a study done in a Bangladeshi population during 2003-2004. ${ }^{10}$ To date, enough data are not available regarding risk factors, useful biochemical markers which can detect premature babies at risk.

Contemporary diagnosis of overt osteopenia has 
been based on characteristic radiological changes ${ }^{11}$. However, the radiological changes of osteopenia are not easily detected until bone mineralization is reduced by at least $20 \% .^{12}$ Dual energy X-ray absorptiometry (DEXA) is considered to reflect most accurately the state of bone mineralization. Because of the rarity of DEXA instruments, a biochemical marker would be a good alternative in resource limited centers. ${ }^{13}$ Serum alkaline phosphatase, serum calcium and serum inorganic phosphates have been suggested to be a way of identifying preterm infant with osteopenia of prematurity. ${ }^{14}$ There is scarcity of evidences that any of the frequently used serum markers are valid marker of osteopenia of prematurity. Most of the authors suggested 3 weeks of life as early period of screening as alkaline phosphatase level rises in all newborns in the first 2-3 weeks of life and increases further if there is insufficient mineral supply. ${ }^{12}$ Hung et al concluded that serum ALP exceeding 700IU/L at 3 weeks post natal age can predict the risk of osteopenia of prematurity. ${ }^{12}$ Backstrom et al showed that high serum alkaline phosphatase activity and low serum phosphate level are the best available screening method for low bone mineral density. ${ }^{13}$ A systematic review compared the results of frequently used serum and urine measurements to the results of number of imaging techniques, which showed that increased urinary calcium concentration, may be a valid biochemical marker, but more research was recommended to confirm this. ${ }^{11}$ Currently, there is neither standard recommendation for screening of osteopenia of prematurity nor data are available describing the usual values of bone markers in preterm Infants. ${ }^{15}$ Scarcity of evidences regarding screening and diagnostic tool might have led premature infants undiagnosed subsequently subject them to the risk of growth failure. Therefore, this study was intended to examine the diagnostic performance of biochemical markers in early detection of osteopenia of prematurity.

\section{Materials and Methods}

This prospective study was conducted in the Neonatal Intensive Care Unit (NICU), Department of Neonatology, Bangabandhu Sheikh Mujib Medical University over a period of 9 months (June 2013 to February 2014). After obtaining informed written consent from parents, a total of 100 neonates were enrolled consecutively. Infants with haemodynamically unstable prematurity, congenital anomalies and suspected congenital bone or muscle disease were excluded. Baseline demographics including gestational age (GA), birth weight (BW), small for gestational age (SGA), gender and perinatal characteristics including place of delivery, mode of delivery, Apgar score at 5 minutes were collected. Birth weight was recorded from labour room or neonatal referral sheet in case of out born infants. Weight at admission was taken by a digital weighing scale (SALTER, Model-914). Gestational age was assessed by LMP, sonographic findings and modified new ballard scoring. Postnatal medical records (respiratory distress syndrome, sepsis, and retinopathy of prematurity, time to full feeding) were recorded.

Blood samples for biochemical tests were done biweekly from 1 week postnatal age onwards up to corrected term age. During study period, these biochemical markers were incorporated into routine biochemical follow-up of premature infants and sample were taken aseptically adjusting with other purposes of venepuncture. Frequency was at least 3 in infants with gestational age $\geq 33$ weeks, 4 in $\geq 31$ weeks, 5 times in $\geq 29$ weeks. Serum alkaline phosphatase activity was measured by colorimetrically by $\mathrm{p}$ nitrophenyle phosphorus acid radical quality method. It was reported in $\mathrm{U} / \mathrm{L}$ and normal reference value for adult was 50-136 U/L. A prefeed blood sample in a plain tube was used. Serum calcium was examined by the complexed cresolephthalein test. A Level of $<7 \mathrm{mg} / \mathrm{dl}$ was taken as low calcium value for the newborn. Serum inorganic phosphate was measured using a direct phosphomolibdate reaction, which can be measured colorimetrically. Normal reference value was taken as 3.0-4.5 mg/dl. Enrolled premature infants underwent for radiologic examination of forearm with portable X-ray machine at corrected term age. Radiologic examination of forearm with portable X-ray machine was performed by single radiographer. Infants were exposed to radiation dose at 50-55 $\mathrm{kVp}$ (kilovolt peak) and $0.5 \mathrm{mAs}$ (milliamp seconds). Diagnostic reference level was $68 \mathrm{kVp}$ / 0.5mAs. Comments on radiographic findings were made by a radiologist who was blinded to laboratory findings. The scoring method of Koo et $\mathrm{al}^{17}$ was used to assess osteopenia. Grade 0 has 
been defined as findings of normal bone, grade 1 as mineral rarefaction, grade 2 as fraying and cupping of the metaphyses and grade 3 as fractures. Osteopenia was considered when there is radiographic evidence of diminished bone density defined as $\geq$ grade 1 . Patients' outcomes including presence of osteopenia, length of hospital stay, gestational age at radiologic examination, gestation were documented.

Study was approved by Institutional Review Board (IRB) of Bangabandhu Sheikh Mujib Medical University. Data were compared between osteopenic and non-osteopenic using Chi Squared test for categorical variables and Independent- $t$ test for continuous variables. Receiver operator characteristics (ROC) curves were used to determine the individual diagnostic performance of biochemical bone markers. The statistical software IBM SPSS Statistics version 19 (SPSS Chicago, IL) was used for the statistical analysis. Results were considered statistically significant at $p<0.05$.

\section{Results}

During the study period, 190 eligible preterm infants $\leq 34$ weeks gestation were admitted in the Neonatal Intensive Care Unit. After taking informed written consent, a total of 100 neonates were enrolled. Three parents denied giving the blood for serum bone biochemical markers estimation and withdrew consent, 9 patients died during study period (6 during hospital stay, 3 after discharge from hospital) and 10 patients did not complete the follow-up and radiological diagnosis. The remaining 78 infants were ultimately retained for analysis. Demographic and perinatal characteristics of studied infants are presented in table I. Of the 78 infants, $36(46 \%)$ developed radiological evidence of osteopenia at corrected term age. Of them, $22(28 \%)$ infants were grade $1,13(17 \%)$ were grade 2 , remaining $1(1 \%)$ was grade 3 osteopenia according to Koo's criteria ${ }^{17}$. Mean gestational age of radiologic detection of osteopenia of prematurity was $39 \pm 1.3$ weeks. According to the gestational age category, osteopenia was significantly more frequent $(22 / 31,71 \%)$ in less than 32 week premature infants in comparison to infants with gestational age $\geq 32$ weeks. Comparison of demographic and clinical characteristics in premature infants who had osteopenia and those without osteopenia at corrected term age is shown in table II. Compared with non-osteapenic infants, infants with osteopenia were significantly smaller ( $p$ value 0.001$)$ and more premature $(p$ value <0.001). Infants with osteopenia suffered from sepsis significantly more frequently than non-osteapenic infants $(p$ value 0.01). Premature infants with osteopenia tended to have significant delay in achieving full feed ( $p$ value 0.001 ) than that of infants without osteopenia. Significant difference was also found in case of need for mechanical ventilation ( $p$ value 0.028 ). Hospital stay was also significantly prolonged in osteopenic preterm infant than that of non-osteopenic infants ( $p$ value 0.001 ).

Table I: Baseline characteristics of enrolled premature infants $(\mathrm{n}=78)$

\begin{tabular}{lc}
\hline \multicolumn{1}{c}{ Variable } & Infants \\
\hline Birth weight(g) & $1384 \pm 223$ \\
Gestational age (weeks) & $31.94 \pm 1.6$ \\
Male & $44(56 \%)$ \\
Inborn & $56(71 \%)$ \\
Hospital delivery & $70(90 \%)$ \\
Cesarean section & $47(60 \%)$ \\
Small for gestational age & $16(20 \%)$ \\
Low APGAR score in 5 & $16(21 \%)$ \\
minutes (<7) & \\
\hline
\end{tabular}

Numerical data are presented as mean $\pm \mathrm{sd}$ and categorical data as percentage $(\%)$

Table II: Demographic and clinical characteristics in osteopenic infants compared with non-osteopenic infants

\begin{tabular}{llll}
\hline \multicolumn{1}{c}{ Variable } & \multicolumn{1}{c}{$\begin{array}{c}\text { Osteopenic } \\
(\mathbf{n ~ 3 6})\end{array}$} & $\begin{array}{c}\text { Non- } \\
\text { osteopenic } \\
(\mathbf{n}=\mathbf{4 2})\end{array}$ & $\boldsymbol{p}$ value \\
\hline $\begin{array}{l}\text { Gestational age } \\
\text { (wks) }\end{array}$ & $31.17 \pm 1.50$ & $32.60 \pm 1.49$ & $<0.001$ \\
$\begin{array}{l}\text { Birth weight(g) } \\
\text { Male (\%) }\end{array}$ & $\begin{array}{l}1295 \pm 189 \\
18(50 \%)\end{array}$ & $\begin{array}{l}1460 \pm 224 \\
23(55 \%)\end{array}$ & 0.001 \\
$\begin{array}{l}\text { Small for } \\
\text { gestational age }\end{array}$ & $9(25 \%)$ & $6(14 \%)$ & 0.30 \\
$\begin{array}{l}\text { Sepsis } \\
\text { Time to full }\end{array}$ & $16(44 \%)$ & $8(19 \%)$ & 0.01 \\
feed (d) & $20 \pm 8.17$ & $4 \pm 6.7$ & 0.001 \\
Need for MV & $6(16 \%)$ & $1(3 \%)$ & 0.02 \\
RDS* & $8(22 \%)$ & $6(14 \%)$ & 0.36 \\
ROP** & $6(16 \%)$ & $5(12 \%)$ & 0.42 \\
$\begin{array}{l}\text { Length of } \\
\text { hospital stay (d) }\end{array}$ & $27 \pm 10.7$ & $18 \pm 9.0$ & $<0.001$ \\
\hline
\end{tabular}

* Respiratory distress syndrome, **Retinopathy of prematurity

Comparison of various biochemical markers of osteopenia of prematurity among osteopenic and non-osteopenic infants are demonstrated in table III. 
Table III: Serum alkaline phosphatase, serum inorganic phosphate, serum calcium in osteopenic infants compared with non-osteopenic infants

\begin{tabular}{|c|c|c|c|}
\hline Variable & $\begin{array}{l}\text { Osteopenic } \\
\text { (n 36) }\end{array}$ & $\begin{array}{c}\text { Non- } \\
\text { osteopenic(n } \\
=42)\end{array}$ & $p$ Value \\
\hline \multicolumn{4}{|c|}{ Serum Alkaline Phosphatase } \\
\hline 1-4 weeks & $224 \pm 48$ & $210 \pm 42$ & 0.23 \\
\hline $5-8$ weeks & $494 \pm 138$ & $274 \pm 60$ & $<0.001$ \\
\hline \multicolumn{4}{|c|}{ Serum inorganic Phosphate } \\
\hline $1-4$ weeks & $3.9 \pm 0.49$ & $4.8 \pm 0.69$ & $<0.001$ \\
\hline 5-8 weeks & $3.2 \pm 0.80$ & $4.6 \pm 0.50$ & $<0.001$ \\
\hline \multicolumn{4}{|c|}{ Serum calcium } \\
\hline $1-4$ weeks & $8.2 \pm 0.31$ & $8.4 \pm 0.51$ & 0.06 \\
\hline 5-8 weeks & $8.1 \pm 0.37$ & $8.2 \pm 0.79$ & 0.7 \\
\hline
\end{tabular}

Biochemical data were available for $255 / 295$ (87\%) of the study population. Of 255 sets of data, biochemical data of 78 infants were available in 1 week of age, 63 data were collected at 3 weeks of age, 60 data were available at 5 weeks of age and 54 data were documented at 7 weeks of age. As biochemical tests could not be done strictly on biweekly basis, biochemical data were summarized in two time period; 1-4 weeks and 5-8 weeks. The proportion of missing biochemical data were similar between the two groups $(26 / 150$ [17.3\%] vs $18 / 100$ [18\%], $p=0.39$ ). Serum alkaline phosphatase level was significantly elevated in 58 weeks of life $(p<0.001)$ in osteopenic infants when compared with non-osteopenic infants. No significant difference in serum alkaline phosphatase level could be demonstrated between two groups in 1-4 weeks of life. Serum inorganic phosphate level was significantly less in infants with osteopenia than without osteopenia both in1-4 weeks $(p<0.001)$ as well as 5-8 weeks of life $(p<0.001)$. No differences were found between osteopenic and nonosteopenic infants regarding serum calcium level in both time periods.

Receiver Operator Characteristic (ROC) curves was constructed to determine individual diagnostic performance at 1 and 3 weeks postnatal age in predicting the occurrence of osteopenia at corrected term age (figure 1).

Predictive accuracy of serum inorganic phosphate was good and statistically significant in comparison to serum alkaline phosphatase and serum calcium in both the curves. Predictive ROC Curve

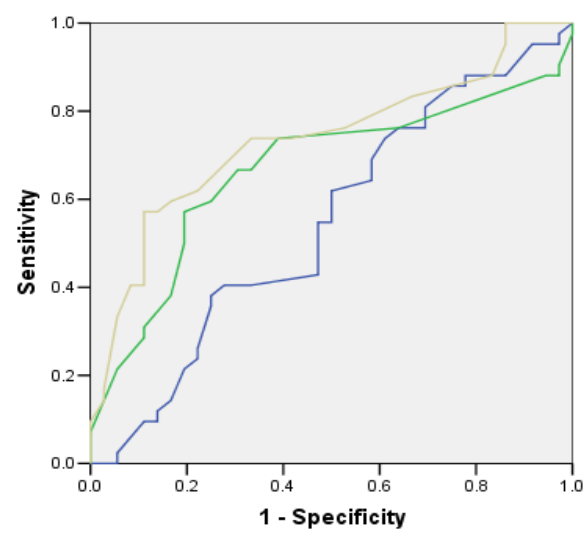

Source of the Curve - alp 7 day ip 7 days
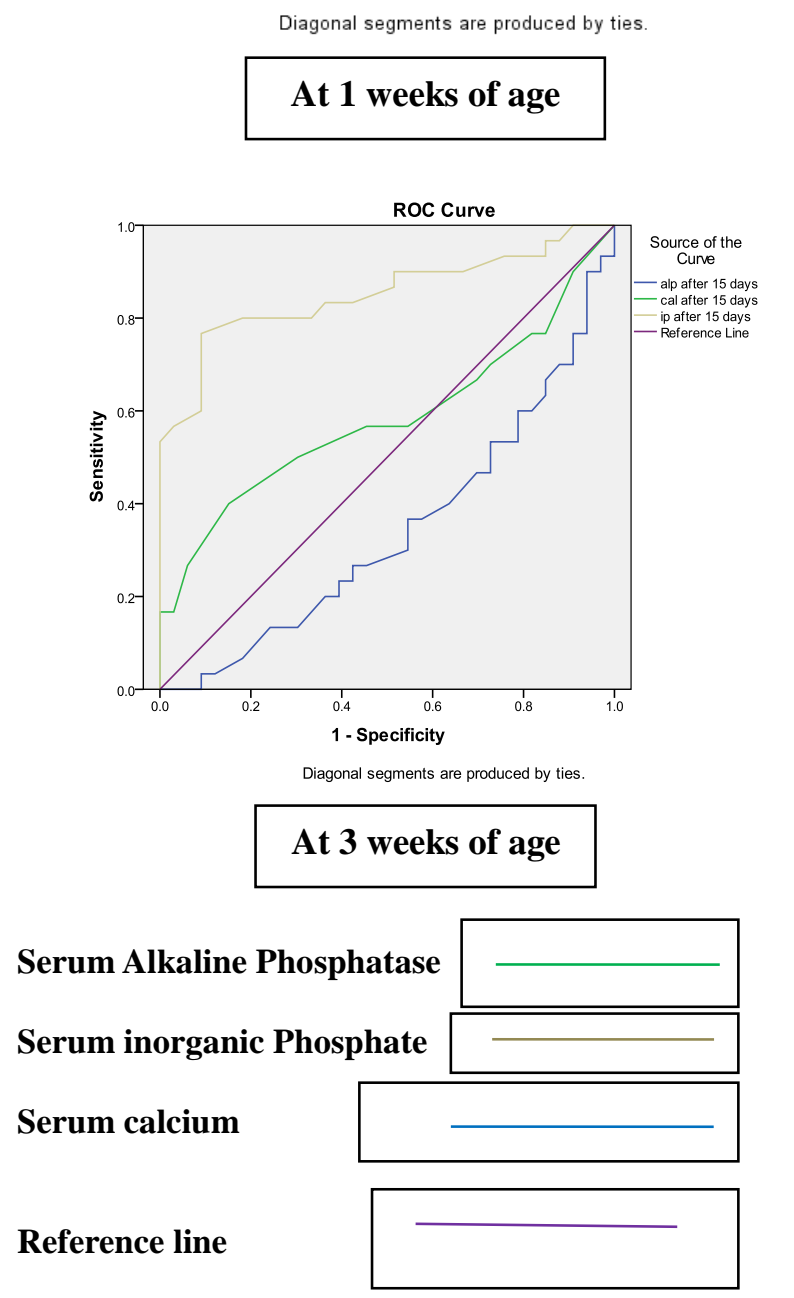

Figure 1: Receiver operator characteristic curves of biochemical markers at 1 and 3 weeks postnatal age in detecting the risk of osteopenia at term age.

accuracy of inorganic phosphate was more in 3 weeks curve as the area under ROC curve for serum inorganic phosphate was $85 \%(p=0.001)$ at 3 weeks of age. At 3 weeks age the areas under 
ROC curve for serum calcium and serum alkaline phosphatase were $58 \%$ and $34 \%$ respectively ( $p 0.29$ and 0.06 respectively). If the cut off value of serum inorganic phosphate was set at 3.6 $\mathrm{mg} / \mathrm{dl}$, then a sensitivity of $86 \%$ and a specificity of $49 \%$ were obtained.

\section{Discussion}

Serum alkaline phosphatase, serum inorganic phosphate and serum calcium have been used to screen, early detection and prediction of osteopenia in the premature infants. In the first 23 weeks of life, with the physiological remodeling, alkaline phosphatase level raises in all newborns and increase further if there is insufficient mineral supply. Newborns receiving sufficient nutrition may have only smaller rises in alkaline phosphatase. ${ }^{18}$ This is the explanation behind the reason of taking biochemical sample at 3 weeks of age by most of the author. In this study, biochemical markers at 3 weeks of age were used to determine predictability of osteopenia at corrected term age.

Receiver operator characteristic (ROC) curves were constructed to examine diagnostic performance of bone marker at 3 weeks of age in predicting the osteopenia at corrected term age. The area under the ROC curve for serum inorganic phosphate was high and statistically significant in comparison to serum alkaline phosphatase and serum calcium. Best cut-off value was obtained at $3.6 \mathrm{mg} / \mathrm{dl}$ yielded sensitivity $86 \%$ and specificity $49 \%$. Backstrom et al attempted to enhance detection rate of osteopenia by combining serum alkaline phosphatase and serum phosphate. ${ }^{13}$ They found that serum phosphate level below $1.8 \mathrm{mmol} / \mathrm{l}$ demonstrate $50 \%$ sensitivity and $95 \%$ specificity at 3 months corrected age. They further combine serum alkaline phosphatase along with serum phosphate and reach $100 \%$ sensitivity and $70 \%$ specificity at $900 \mathrm{U} / \mathrm{l}$ and $1.8 \mathrm{mmol} / \mathrm{l}$ values respectively. Yi-Li Hung et al found striking dissimilarity that in spite of significant difference in the level of the serum phosphorus between osteopenic and non-osteopenic infants at 3 weeks of age, they could not demonstrate predictive accuracy of serum phosphate. ${ }^{12}$ While comparing diagnostic performance of serum alkaline phosphate, serum inorganic phosphate, serum calcium at 3 weeks of age, predictive accuracy of serum inorganic phosphate was considerably smaller than those of serum alkaline phosphatase according to their study findings. They concluded that serum ALP concentration exceeding $700 \mathrm{IU} / \mathrm{L}$ at 3 weeks post-natal age can predict the risk of osteopenia in preterm infants.

The result of this study showed, serum alkaline phosphatase level was significantly elevated only in 5-8 weeks of life in case of osteopenic infants when compared with non osteopenic. There was no significant difference in serum alkaline level between two groups in 1-4 weeks of life, suggesting that serum ALP level is not a good marker of osteopenia in first 4 weeks of life. Serum inorganic phosphate level was significantly less in osteopenic infants than nonosteopenic group in first two months of life, indicating that a persistently low phosphate level contributes to the development of osteopenia of prematurity. A fall in plasma phosphate concentration has been described in very low birth weight infants who developed disturbances in the mineralization, especially among those receiving human milk. ${ }^{13,16}$ This might be the explanation of the finding regarding changes in the serum phosphate in the present study. A retrospective study of 230 conducted by Sreekanth et al demonstrated similar findings with that of present study with the exception that they have investigated biochemical markers among extremely low birth weight population. ${ }^{3}$ To date, role of biochemical markers in detection of osteopenia of prematurity are questionable. Utility of serum alkaline phosphatase and serum phosphate in the detection of osteopenia of prematurity were challenged by $\mathrm{J}$ Faerk et al as they could not demonstrate any association between bone mineral content and mean alkaline phosphatase and serum phosphate. ${ }^{14}$ The role of urinary calcium and phosphorus levels were investigated along with serum calcium and phosphorus levels by catache and Leone. ${ }^{19}$ They argued that serum calcium and phosphorus levels are not good markers in early detection of mineral deficiency, rather urinary calcium level may be helpful in early detection of mineral deficiency.

In this study of a recent cohort of premature babies', 46\% (36/78) had radiological evidence of osteopenia at corrected term age. The incidence is similar to that has been previously 
reported in literature. In 1989, Koo et at ${ }^{17}$ observed $30 \%$ incidence of fractures/rickets in first 1 year of life while following 78 preterm infants. Mitchell et al ${ }^{15}$ selectively screened 32 extremely low birth weight infants with ALP level > $800 \mathrm{IU} / \mathrm{L}$ for radiological rickets. All had evidence of osteopenia and 18/32 (56\%) had radiological rickets. In a recent study by $\mathrm{Yi}-\mathrm{Li}$ Hung et al concluded that osteopenia of prematurity among $\leq 34$ weeker was $39 \%$. ${ }^{12}$ A retrospective review by Burm seok et al between 2009 and 2011 revealed 43\% (112/258) incidence of osteopenia. ${ }^{20}$

During the study period radiology was the only tool to make definitive evidence of osteopenia. So, radiation hazard of X-ray could not be avoided in studied infants. In standard radiograph, the bone changes of osteopenia of prematurity are a late sign as these appear only after $20-30 \%$ of bone mineral content is lost. So, it was possible to miss the premature infants with mild osteopenia.

\section{Conclusion}

Radiologic evidence of Osteopenia was $46 \%$ among premature infants gestational age $\leq 34$ weeks. Low serum inorganic phosphate at around 3 weeks of life can be used as a predictor of osteopenia of prematurity. Future research is recommended to find out a reliable investigation tool to make correct and early diagnosis.

Acknowledgment: The Authors gratefully acknowledge the sincere cooperation of the parents as well as the contribution of all doctors, staffs of the department of neonatology, obstetrics \& gynaecology and the department of radiology of BSMMU

\section{References}

1. Blencowe $\mathrm{H}$, Cousens $\mathrm{S}$, Oestergaard $\mathrm{M}$ et al. National, regional and worldwide estimates of preterm birth rates in the year 2010 with time trends since 1990 for selected countries: a systematic analysis and implications. The Lancet. 2012; 379 (9832): 2162-72.

2. Linda L Wright, Betty R Vohr, Avroy A Fanaroff. Perinatal-Neonatal Epidemiology. Perinatal, mortality and morbidity information for infants born in the National Institute of child Health and Human Development (NICHD) Neonatal Research Network from 1997 to 2000. Avery's disease of the Newborn $8^{\text {th }}$ ed. Philadelphia. Elsevier Saunders; 2004:

3. Viswanathan S, Khasawneh W, McNelis K, et al. Metabolic Bone Disease: A Continued Challenge in Extremely Low Birth Weight Infants. J Parenter Enteral Nutr. 2014 ; 38(8):982-90.

4. Fewtrell M. Early nutritional predictors of long-term bone health in preterm infants. Curr Opin in Clin Nutr and Metab Care. 2011; 14(3): 297-301.

5. Horsman A, Ryan SW, Congdon PJ, Truscott J G and James J R. Osteopenia in extremely low birth weight infants. Archives of Disease in Childhood. 1989; 64: 485-8.

6. Crofton PM, Shrivastava A, Wade JC, et al. Bone and collagen markers in preterm infants: relationship with growth and bone mineral content over the first 10 weeks of life. Pediatric Research. 1999; 46: 581-7.

7. Lyon AJ, McIntosh N, Wheeler K, Williams JE. Radiological rickets in extremely low birth weight infants. Pediatr Radiol. 1987; 17(1): 56-8.

8. Vachharajani AJ, Mathur AM, Rao R. Metabolic Bone Disease of Prematurity. Neo Reviews. 2009; 10: e 402-e411.

9. Bozzetti V, Tagliabue P. Metabolic bone disease in preterm newborn: an update on nutritional issue. $\mathrm{J}$ Pediatr. 2009; 35(1): 20.

10. Afroze N. Osteopenia in premature infants and effect of calcium and phosphorus supplementation on it. FCPS dissertation, Bangladesh College of Physician and Surgeon, Dhaka, January 2007.

11. Visser F, Sprij A J, Brus B. The validity of biochemical markers in metabolic bone disease in preterm infants a systematic review. Acta Paediatrica. 2012; 101(6): 562-568.

12. Hung YL, Chen PC, Jeng SF et al. Serial measurements of serum alkaline phosphatase for early prediction of osteopenia in preterm infants. $\mathrm{J}$ Paediatr Child Health. 2011; 47(3): 134-9.

13. Backstrom MC, Kouri T, Kuusela AL, et al. Bone isoenzyme of serum alkaline phosphatase and serum inorganic phosphate in metabolic bone disease of prematurity. Acta Paediatrica. 2000; 89(7): 867-873.

14. Faerk J, Peitersen B, Petersen S, Michaelsen K F. Bone mineralisation in premature infants cannot be predicted from serum alkaline phosphatase or serum phosphate. Arch Dis Child Fetal Neonatal Ed. 2002; 87: 133-136.

15. Mitchell SM, Rogers SP, Hicks PD, Hawthorne KM, Parker BR and Abrams SA. High frequencies of elevated alkaline phosphatase activity and rickets exist in extremely low birth weight infants despite 
current nutritional support. BMC Pediatrics 2009; 9: 47.

16. Backstrom MC, Kuusela AL, Maki R. Metabolic bone disease of prematurity. Ann Med. 1996; 28 (4): 275-82.

17. Koo WW, Gupta JM, Nayanar VV, Wilkinson M and Posen S. Skeletal changes in preterm infants. Archives of Disease in Childhood. 1982; 57: 44752 .

18. Tinniton JR, Embleton DN. How to use... alkaline phosphatase in neonatology. Arch Dis Child Educ Pract Ed. 2012; 97: 157-163.
19. Catache M, Leone CR. Role of plasma and urinary calcium and phosphorus measurements in early detection of phosphorus deficiency in very low birth weight infants. Acta Paediatrica. 2003; 92 (1): 76-80.

20. Oh BS, Choi JS, Kim YN, Song ES, Choi YY. Usefulness of serum alkaline phosphatase in predicting osteopenia and rickets in very low birth weight infants. J Korean Soc Neonatol 2012; 19 (4): 229-236. 